\title{
Civilisations
}

Revue internationale d'anthropologie et de sciences

humaines

$63 \mid 2014$

L'Ancestralité revisitée

\section{Chanter les ancêtres pour enraciner les vivants chez les Jóola de Casamance (Sénégal)}

Jean Baptiste Valter Manga

\section{OpenEdition \\ Journals}

Édition électronique

URL : http://journals.openedition.org/civilisations/3730

DOI : 10.4000/civilisations.3730

ISSN : 2032-0442

\section{Éditeur}

Institut de sociologie de l'Université Libre de Bruxelles

\section{Édition imprimée}

Date de publication : 30 septembre 2014

Pagination : 163-178

ISSN : 0009-8140

Référence électronique

Jean Baptiste Valter Manga, «Chanter les ancêtres pour enraciner les vivants chez les Jóola de

Casamance (Sénégal) », Civilisations [En ligne], 63 | 2014, mis en ligne le 30 septembre 2018, consulté

le 01 mai 2019. URL : http://journals.openedition.org/civilisations/3730 ; DOI : 10.4000/

civilisations.3730 


\title{
Chanter les ancêtres pour enraciner les vivants chez les Jóola de Casamance (Sénégal)
}

\author{
Jean Baptiste Valter MANGA
}

\begin{abstract}
Résumé : Cet article porte sur la place des ancêtres dans l'organisation sociale d'un sous-groupe des Jóola de Basse Casamance au Sud Ouest du Sénégal. S'il est difficile de parler d'un "culte » dont ils seraient l'objet, les ancêtres restent des références essentielles à partir desquelles s'identifient les individus et s'articulent les rapports sociaux. Le processus d'ancestralisation, organisé par les agnats du défunt, se fait notamment par la mise en chant des qualités de celui-ci. De leur composition aux divers contextes d'exécution ces chants, appelés en jóola kuhuluy, constituent un patrimoine lignager et villageois dont la mobilisation fait jouer aussi bien les rapports de parenté, le droit au sol etc. Plus qu'un honneur à leur mémoire, l'évocation des ancêtres sert en même temps à réaffirmer l'ancrage de leur descendance dans la communauté villageoise. Après une période de crise dans la composition et la transmission due à l'influence des religions universalistes (Christianisme et Islam) et à la forte migration, le répertoire de chants ancestraux recouvre un intérêt manifeste dans ces milieux (celui des convertis et des émigrés) qui l'avaient pendant un temps négligé. Ce regain d'intérêt qui fait fi des appartenances religieuses montre que les ancêtres redeviennent - pour ceux qui ont pris de la distance géographiquement ou par rapport aux croyances - un moyen de renouer avec les origines.
\end{abstract}

Mots-clés : ancêtres, Casamance, Jóola, répertoire de chants, funérailles, légitimation, transmission.

\begin{abstract}
This article focuses on the role of ancestors in the social organization of a subgroup of Joola in the Lower Casamance region of southwestern Senegal. The ancestors in this case, while not comprising a "cult" of ancestors as such, remain key reference points from which individuals identify and articulate social relationships. The process of ancestralization organized by the agnates of the deceased is performed through the creation of songs exalting the qualities of the latter. The composition and the various contexts of the execution of these songs, called kuhulun in Joola, highlight both family and village histories as well as local cultural heritage, and whose mobilization may put into play kinship relations, land rights etc. More than homage to the memory of the ancestors, evoking their memory simultaneously serves to reaffirm and anchor their descendants in the village community. After a period of crisis in the composition and transmission of these songs due to the influence of universalist religions (Christianity and Islam) and significant migration, the repertoire of ancestral songs has reinvigorated the interest on the part of these groups (converts and emigrants) for their culture which had, for a time, been neglected. This renewed interest, which ignores religious affiliation, shows that the ancestors are becoming - for those who have become distant geographically of spiritually - a way to reconnect with their origins.
\end{abstract}

Keywords: ancestors, Casamance, Jóola, repertoire of songs, funerals, legitimatization, transmission. 


\section{Introduction}

La place qu'occupent les ancêtres dans l'organisation politico-religieuse des sociétés africaines a été diversement abordée par les anthropologues. Du culte des ancêtres vu comme la marque définitive de la religion primitive (Spencer, Tylor, Frazer) dont l'étude pouvait conduire à une meilleure compréhension de l'évolution de la religion en particulier et du social en général, on est passé, notamment avec Fortes (Fortes 1959), à une vision plus fonctionnaliste mettant l'accent sur les rapports de pouvoir. À partir des recherches conduites chez les Tallensi du Ghana, Fortes relève que ceux-ci ont un culte des ancêtres non parce qu'ils craignent les morts, ou qu'ils croient en l'immortalité de l'âme, mais parce que leur structure sociale l'exige (Fortes 1959 : 66).

Le débat prend une nouvelle dimension avec la publication en 1971 par Igor Kopytoff d'un article intitulé « Ancestors as elders in Africa ». Pour Kopytoff, la question de l'autorité de la personne, qu'elle soit déjà ancestralisée ou encore en vie, n'était qu'une préoccupation des chercheurs et avait peu de pertinence pour les Africains. Les chercheurs occidentaux auraient aussi exagéré la dimension surnaturelle des ancêtres. Cet article a suscité diverses critiques (Brain 1975 ; Mendonsa 1976 ; Calhoun 1980).

Plus récemment, John McCall (1995 : 258), remarquant l'importance des ancêtres dans la vie quotidienne de diverses populations ouest africaines, a suggéré une meilleure prise en compte de leur rôle dans la construction et la reproduction d'une conscience historique et identitaire. Une bonne compréhension du rôle des ancêtres passerait par une relativisation des limites imposées par le « culte » et la « religion » qui définissent traditionnellement l'enquête de terrain sur ce sujet.

Admettre que le religieux est aussi lieu du politique conduit à prendre ensemble, sans les opposer systématiquement, les pratiques religieuses et les stratégies politiques. Et de ce fait, qu'ils soient objets de culte ou non, les ancêtres peuvent devenir une ressource mobilisable dans les rapports sociaux, et l'attention autour de leur mémoire, un repère pour « localiser » l'autorité (Gable 1996).

En élargissant la perspective, c'est-à-dire en considérant de manière plus générale les funérailles, qui sont le cadre du processus d'ancestralisation, diverses études dans le contexte africain ont montré comment elles institutionnalisent la mise en scène des rapports de pouvoir et de dépendance (Vidal 1986 ; Tonda 2000). Les funérailles en Afrique sont, comme le remarquent Jindra et Noret, les meilleures occasions pour (re) produire et (re)faire aussi bien les rapports de solidarité, de hiérarchie, d'alliance, que les conflits (Jindra et Noret 2011).

Ces questions qui portent sur le rôle des ancêtres dans les rapports sociaux sont abordées ici au prisme de chansons composées à leur intention. Il s'agira de montrer comment, chez les Jóola d'Oussouye, la fabrication de chants pour les morts et le fait de les exécuter sont une forme d'action dans le jeu social.

Après quelques éléments contextuels, je présenterai les différentes occurrences du mot kuhuluy, qui désigne l'ensemble des chansons dédiées aux ancêtres. Cette analyse terminologique permettra d'éclairer les connections entre le domaine culturel, et les pratiques liées à l'attribution du nom ou à la territorialisation. La présentation du processus d'élaboration du répertoire de chants ancestraux et des usages dont il fait l'objet, mettra en exergue les logiques de pouvoir, de genre, etc. Dans la dernière partie, je montrerai comment les différents changements sociaux induits par les phénomènes de 
mondialisation et de globalisation offrent de nouvelles perspectives à l'usage des chants des ancêtres et comment les nouvelles générations se les approprient effectivement.

\section{Oussouye : un royaume de Casamance}

La Basse Casamance se situe dans la zone géographique des côtes ouest africaines comprise entre les îles du Saloum et la Sierra Leone. Appelées « Rivières du Sud »" pour les francophones ou «Upper Guinea Coasts » du point de vue des Anglais, ces côtes sont occupées par diverses populations dont beaucoup pratiquent une riziculture inondée à partir parfois d'un aménagement très ingénieux de la mangrove (Pélissier 1966). Les populations appelées Jóola sont réparties sur la partie de cet ensemble comprise entre le Fleuve Gambie et le Rio Cacheu en Guinée-Bissau.

$\mathrm{Au}$ moment de l'irruption de la colonisation française, ces populations étaient réunies en de petits royaumes plus ou moins autonomes se réclamant d'un même centre : la royauté de Karuhay, village situé à la frontière entre le Sénégal et la Guinée-Bissau. Les royaumes de la rive nord de la Casamance ont tous disparu avec la colonisation. Certains de la rive sud par contre se sont maintenus et servent encore de cadre de référence en termes de pratiques rituelles, d'organisation sociale, etc. (Thomas 1959 ; Girard 1969 ; Baum 1999 ; Tomas 2005). Le royaume d'Oussouye dont il est question dans cet article est le plus vaste de ces structures précoloniales ; il est composé de dixsept villages.

En choisissant de faire du village d'Oussouye un chef-lieu de province en 1920, l'administration coloniale faisait ainsi le choix de s'installer dans la même cité que le roi dont les pouvoirs avaient déjà été drastiquement réduits (Roche 1985). Cette installation ouvre la voie au Christianisme et à l'Islam. L'Église catholique installe une mission en 1926. Les conversions, qui ne manquent pas de créer des tensions au sein des lignages et villages, sont assez timides au début. Toutefois, le nombre de catholiques augmente rapidement vers 1980, surtout parmi les jeunes et ceux qui migrent vers les villes sénégalaises, ce qui se traduit souvent par des pratiques religieuses différentes au sein des familles. Quant à l'Islam, bien que l'installation de villages musulmans aux abords d'Oussouye soit antérieure à l'arrivée du christianisme (Baum 1999; Méguelle 2012), les conversions de Jóola sont restées rares encore aujourd'hui car l'Islam dans cette région est avant tout la religion des commerçants ou migrants sahéliens. La majorité de la population adulte reste ainsi attachée à la religion « traditionnelle ».

La naissance au début des années 1980 d'un mouvement indépendantiste et la situation de guerre qui s'en est suivie avec l'État sénégalais a occasionné, particulièrement durant les années 1990, de grands mouvements de populations (Foucher 2002 ; Marut 2010). Le cycle des grands rituels initiatiques a aussi été affecté de même que la royauté d'Oussouye dont le siège est resté vacant entre 1984 et 2000. En 2000, l'intronisation d'un roi est présentée sur place comme le " retour de la tradition », et la popularité de ce roi ne cesse depuis lors de grandir aussi bien auprès des populations locales que de l'administration sénégalaise, des ONG, des milieux touristiques etc.

La matière qui compose cet article est essentiellement issue des enquêtes que j'ai menées, dans le cadre de recherches doctorales entre 2009 et 2013, à Oussouye afin

1 Cf. M.-C. Cormier-Salem, J.-P. Chauveau et S. Bouju, 1999. 
de comprendre la problématique du « retour de la royauté » dans cette région et à ce moment de l'histoire de la Casamance et du Sénégal.

\section{Terminologie}

Le mot Kuhuluy désigne trois choses : un autel, un interdit d'évocation de nom et un répertoire de chants.

L'autel kuhuluy est intimement lié à la concession du groupe patrilinéaire, appelée en jóola 'hank', dont il matérialise la permanence. C'est en effet au sein de l'espace de la concession, qui apparaît sur la scène sociale et rituelle comme une personne morale (Journet-Diallo 2007 : 78) que l'autel kuhuluy est toujours situé. Il s'agit d'une cour, généralement circulaire, formée par un ensemble de résidences familiales construites les unes à côté des autres.

Même si elle tend à disparaître de nos jours ${ }^{2}$, la concession garde toute sa valeur rituelle et politique. C'est là que se tiennent les rassemblements importants du lignage. Les grandes étapes de la vie d'un individu y sont marquées, servant comme une porte d'entrée et de sortie ou mieux, de changement d'état ${ }^{3}$. Les enfants y sont présentés à la naissance, les jeunes filles lors du départ en mariage, les jeunes hommes au moment des initiations et les défunts avant d'aller reposer dans la parcelle de terre destinée au lignage dans le cimetière du village ou du quartier ${ }^{4}$. La concession est véritablement le lieu où chaque individu est comme ancré à la terre. De la naissance à la mort, toute personne est membre d'un lignage hank qui lui sert de point d'ancrage et d'existence sociale, lui donnant de ce fait droit à la terre, c'est-à-dire à celle liée à son lignage (Adler $2000: 75$ ).

Lors du rite de présentation d'un nouveau-né à l'autel Kuhuluy, les anciens du lignage et les parents de l'enfant annoncent la venue d'un nouvel individu et demandent que son double animal «ewuwum » soit associé à ceux des autres membres du lignage et qu'il y soit protégé. La non-exécution de ce rite pour un enfant signifie aussi sa nonreconnaissance. À l'issue de ce rite, appelé « kasoboo », que l'on pourrait traduire par « demander des faveurs », il est procédé à la dation du nom à l'enfant.

À la mort, l'on y revient pour dire « untel n'est plus des nôtres, conduisez-le dans l'au-delà et qu'il soit avec ceux de son lignage ». Ce rite qui a lieu généralement six jours après le décès est appelé « kalik».

Lorsque quelqu'un meurt en dehors du village, en ville par exemple, le rite « kalik» est précédé d'un autre appelé « hutooñ » (accompagner). Le soir, quand la nuit tombe,

2 Une installation solitaire, souvent loin de la concession d'origine, est privilégiée par les nouvelles générations. Et les incendies répétés ont contraint beaucoup à renoncer à ce mode d'habitat. Toutefois, même lorsqu'il n'y a plus de maison, hank est le lieu incontournable des grands événements lignagers.

3 Je préfère parler de changement d'état parce qu'en réalité l'appartenance à la concession hank paraît définitive. Les femmes par exemple, qui pour des raisons d'alliance matrimoniale « quittent " la concession y reviennent régulièrement pour diverses raisons, gardant toutes les prérogatives d'agnates. Les morts quant à eux, hormis les bébés qui décèdent avant d'avoir eu une dent, y trouvent leur mémoire inscrite à travers les chants du répertoire familial dédié aux défunts.

4 Dans la zone d'Oussouye, les cimetières sont toujours en forêt. Un village peut, en fonction de sa taille, en avoir un ou plusieurs. Toujours est-il que chaque quartier y a son lopin de terre pour enterrer ses défunts. 
les hommes se rendent à la lisière du village, en fonction de l'endroit par où est arrivé le corps (ou ceux qui « ramènent le deuil ${ }^{5}$ » lorsque l'enterrement a eu lieu ailleurs). Une fois les hommes rassemblés, le défunt est appelé d'un cri comme s'il était loin. Alors commence une longue procession qui entonne les chants des ancêtres du lignage jusqu'à l'autel kuhuluy. Il n'y a pas de sacrifice à cette occasion.

Avant le rite « kalik », qui clôture la période des funérailles, le prénom du défunt peut être évoqué. Mais, passé ce rite, le prénom devient kuhuluy, c'est-à-dire qu'il ne doit plus être prononcé. Si par mégarde quelqu'un prononce le prénom d'un défunt, il lui est immédiatement rétorqué : "kuhuluy ». Une telle évocation peut même être vue comme une provocation s'il s'avère que c'est fait sciemment. On peut demander aussi à celui qui prononce le prénom d'un défunt : "où l'as-tu croisé ? ». Autrefois, et ce jusqu'à la fin des années 1970, après le rite de clôture des funérailles toute image (photo) du défunt était détruite. Pour évoquer un défunt, l'on passe par les rapports de parenté ou d'alliance : le père, la mère, le frère ou la sœur, l'époux ou l'épouse, le beau-frère d'untel.

La disparition du nom laisse place aux chants qui ont été composés pour le défunt après sa mort et qui sont sa nouvelle identité. En effet, le lendemain de l'enterrement d'un individu (sauf pour les bébés), les femmes du village se réunissent dans la concession familiale du lignage (hank) pour apprendre les chants composés pour le défunt. Ainsi, pendant six jours, elles passeront les journées ou les après-midi à cet exercice. Généralement, chaque femme apporte sur place du matériel qui lui permet de faire un petit travail au cours des répétitions de chants : tissage, couture, tri de riz ou noix quelconques, etc.

Cette activité de composition et d'apprentissage de chants de défunts est appelée «bulako » (le fait de rester), les femmes passent en effet des heures assises à chanter. Son organisation est assurée par les agnates du défunt. La composition de chants pour leur frère, cousin ou oncle décédé est une des nombreuses tâches qu'elles assurent dans la maison du défunt, où elles deviennent temporairement les véritables gestionnaires (Journet-Diallo 2007) : durant les six jours après l'inhumation, ce sont elles qui composent les chants et qui les enseignent aux autres femmes.

Les différentes occurrences du mot kuhuluy, comme autel, interdit d'évocation du nom et répertoire de chants, renvoient à une seule et même réalité : le changement d'état du défunt. L'individu, de son vivant, reste fondamentalement marqué par le prénom qui lui a été attribué six jours après sa naissance sur l'autel des ancêtres. Chez les Jóola, les premiers jours qui suivent la naissance d'un enfant sont entourés de secrets gardés par les femmes. Du sexe de l'enfant à son identification par un prénom, elles lèvent petit à petit un coin du voile, tenant les hommes à l'écart jusqu'à ce que l'enfant soit présenté à l'autel des ancêtres. Comme le note justement Odile Journet-Diallo :

jusqu'à l'apparition de certains signes, le doute plane tant sur le caractère humain de l'enfant que sur son désir de rester ici-bas. La naissance biologique ne suffit pas à séparer le nourrisson du monde invisible avec lequel il garde tant d'affinités [...] Le moment de la dation du nom est l'un des moments

5 L'expression « ramener le deuil » désigne l'arrivée au village des personnes qui ont participé aux obsèques d'un des leurs inhumé en dehors de la localité. 
privilégiés de l'ancrage du nourrisson dans la société humaine.

(Journet-Diallo $2001: 51-52$ )

Le choix d'un prénom pour un nouveau-né était ${ }^{6}$ fonction de certains critères liés entre autres aux habitudes d'un des parents durant la période de grossesse ou à un événement mémorable à la naissance. Un enfant dont la mère aimait se rendre dans une localité, par exemple Oussouye, se verra appelé Ampa Oussouye pour un garçon ou Aju Oussouye si c'est une fille. Un enfant qui naîtrait le jour où les initiés entrent en réclusion pourrait s'appeler « Kalubo », c'est-à-dire « commencement », sous-entendu de l'initiation.

Dans tous les cas, l'individu est lié à son milieu social par le nom qui lui est donné. Les personnes qui portent par exemple des noms en lien avec une initiation deviennent des repères temporels qui permettent d'apprécier les écarts d'une initiation à une autre. C'est ce lien au temps ou à l'environnement social que rompt l'interdit de prononcer le nom d'un défunt (Kuhuluy). L'autel des ancêtres est l'instance qui marque ce changement de statut de l'individu, à la naissance comme à sa mort. Le répertoire de chants (kuhuluy) se substitue ainsi au nom pour créer de nouveaux liens, avec les ancêtres d'une part et les vivants d'autre part.

\section{Le chant kubuluy}

Le chant kuhuluy est composé principalement d'un surnom «kasal» (pl.usal) et de faits marquants de la vie d'une personne. Kasal pourrait se traduire par louange. Il est un surnom louangeur donné à un individu pour marquer divers points positifs de sa vie. Le surnom ou les surnoms peuvent être donnés à une personne (homme ou femme) dès la naissance (selon les premières réactions de l'enfant) ou au cours de la vie en fonction de ses exploits. On entend souvent dire « anaine kasal» (à l'homme, le surnom). Cette expression montre qu'un homme sans surnom n'en est pas vraiment un. Le surnom est même souvent plus utilisé que le prénom.

Dans le chant kuhuluy, le surnom ( $k a s a l$ ) est associé à des faits liés aux qualités de la personne. Qualités physiques : force, beauté, habileté, rapidité etc. Qualités morales : justice, générosité etc. ; la richesse y occupe également une place importante.

À la mort d'un individu, ses agnates se concertent et font une évaluation de sa vie afin de relever toutes ses qualités et les événements marquants du lignage ou du village auxquels il a pris part de façon remarquable. Ne sont retenus que les aspects positifs de la vie du défunt. Le négatif est passé sous silence.

Ces paroles (usal et faits) sont mises en musique par ces femmes. La composition des chants s'appelle « kajuul ukijen » (créer des chants). L'air du chant peut être créé de toute pièce tout comme il peut être puisé dans le vaste répertoire de chant kuhuluy de toute l'aire culturelle jóola. Il n'est pas rare que les femmes qui voyagent loin (en Guinée-Bissau par exemple) rapportent des airs tout à fait nouveaux qu'elles adaptent à leur manière.

6 Aujourd'hui, les noms sont choisis dans divers répertoires selon la religion des parents. Des noms «jóola » continuent tout de même à être donnés aux enfants, souvent comme second prénom. 
Quand un homme a une idée de chant pour un défunt, il va la confier à l'une des agnates qui se chargera de l'apprendre aux autres. Cependant, lors de la période de fabrication et d'apprentissage des chants, si les hommes constatent qu'un chant n'est pas juste, ils le font remarquer afin de l'ajuster ou de l'améliorer. L'apprentissage se fait en deux chœurs qui se répondent à une cadence très lente. Les erreurs de ton ou de paroles sont corrigées au fur et à mesure. Chaque défunt a plusieurs chants, parfois une dizaine. En voici un à titre d'exemple :

1. Wooe woo wo oe Aelefook owowoe

Wooe woo woo e Aelefook owowoe

2. Ebaakiit kufullo jiwanul ukuujaal

De jeunes garçons sont arrivés! Faites descendre et luttons

3. Aelefook alulumoo lokaale

Aelefook "un blanc" que nous avons.

«Aelefook» est le surnom de celui pour qui ce chant a été composé. Le surnom lui-même est composé du verbe kawel (faire du bruit) et du mot efook (quantité de terre soulevée par un cultivateur avec sa pelle-kajand $u^{7}$ ). Il faut être suffisamment habile à remuer la terre pour qu'à chaque poussée de la pelle-kajandu, une masse de terre s'élève et fasse du bruit en retombant. Ce surnom s'applique à un bon cultivateur.

La deuxième phrase évoque l'arrivée de jeunes garçons et demande aux jeunes du village d'Aelefook, particulièrement ceux de sa classe d'âge, de se tenir prêts pour la lutte. Jiwanul que j'ai traduit dans ce chant par « faites descendre » renvoie à ce que font les jeunes avant d'aller lutter. Les accoutrements pour les danses préparatoires et la lutte sont souvent accrochés au toit de la maison familiale ou de la chambre des jeunes garçons. Lorsqu'une séance de lutte avec un autre village est annoncée, chaque jeune fait descendre son paquetage pour s'y préparer.

La troisième partie dit qu'Aelefook est un «blanc » pour les siens. L'usage du qualificatif «blanc » est fréquent dans les chants jóola. Le terme fait référence à l'homme blanc certes, mais plus largement à la personne et à certaines structures issues de la rencontre coloniale. C'est ainsi par exemple que tout ce qui est administratif est encore considéré comme des structures de « blancs ». Le blanc dans ce chant signifie l'ouverture à la nouveauté.

Ce chant met en lumière les diverses qualités d'Aelefook. D'abord sa force et surtout son habileté dans le travail des rizières, qui est l'une des activités les plus valorisantes dans ce milieu, rassurant ses agnates que sa famille ne peut craindre la misère.

D'autre part, ses qualités de bon lutteur font que le village peut aussi compter sur lui pour ne pas perdre la face à l'occasion des luttes inter-villageoises qui sont en réalité des occasions de mise à l'épreuve de l'unité du village et de sa capacité à tenir tête à un adversaire.

Enfin, Aelefook est un homme qui sait tirer profit de l'offre de son temps. La migration saisonnière a été très tôt assez importante chez les Jóola d'Oussouye. Après les travaux rizicoles, les hommes pouvaient se rendre très loin de leur village (Dakar, Kolda, Thiès) pour la récolte du vin de palme. Cette activité permettait de disposer de ressources financières pour payer les impôts et surtout pour équiper sa maison en

7 Kajandu est une pelle avec un long manche que les Jóola utilisent pour cultiver. 
produits manufacturés. Le fait qu'il est dit dans le chant qu'Aelefook est un blanc montre à la fois son ouverture au nouveau monde moderne et son courage au travail.

\section{Archivage et usages du chant kubuluy}

Les chants appris par les femmes sont enseignés aux hommes, singulièrement lors des rites de clôture du deuil (kalik) au sein de la concession du défunt. Certains hommes, appelés en Jóola kukita (sing. akita), aux capacités de mémorisation reconnues, approfondissent la connaissance des chants et leur intégration dans le répertoire familial. Le nom Akita, vient de la racine «kit» qui renvoie à l'idée de commencement. Kakit veut dire commencer quelque chose ${ }^{8}$.

Akita, celui qui débute le chant et déroule le répertoire lors des rassemblements, est un homme qui a un don particulier de mémorisation et de classification. Cette qualité personnelle ne se transmet pas au sein de structures familiales particulières. La performance dépend de la mémoire et de la façon dont l'acteur est capable de la mobiliser en toutes circonstances. Le pari est d'avoir en mémoire un répertoire important, d'être capable de remonter les généalogies le plus loin possible. Il s'agit aussi, et peut être surtout, de bien connaître l'enchaînement à suivre en fonction des lignages, du village et des événements. En principe, chaque quartier a son chantre (Akita). Ils peuvent être plusieurs à assurer ce rôle, tour à tour ou ensemble.

Les moments et lieux d'exécution du répertoire kuhuluy sont très divers. Il est fréquent que tout seul, en travaillant par exemple, quelqu'un se mette à fredonner les chants de ses ancêtres. C'est l'un des moyens privilégiés pour entretenir la mémoire, ne serait ce que celle de sa famille. L'exécution individuelle d'un répertoire familial permet aussi une identification au loin de celui qui chante.

L'exécution collective du répertoire kuhuluy est plus formalisée. Le répertoire lignager et villageois est profondément revisité lors des funérailles : la veillée funèbre (humoo) et la danse qui précède l'inhumation (ñikul). Il l'est d'abord par les femmes qui assurent la première partie des danses funéraires en début d'après-midi suivi par les hommes en fin d'après-midi. Les chants sont rythmés par des calebasses pour les femmes et les tam-tams funéraires pour les hommes. Sont chantés en premier les ancêtres du lignage du défunt suivis des autres défunts du village.

Les femmes ne vont pas au cimetière le jour de l'enterrement. Elles s'y rendent toutefois tôt le lendemain matin pour voir la tombe « kajuk yuyaak». Cette visite au cimetière consiste à observer l'état de la tombe et à mieux la recouvrir au cas où des bêtes auraient tenté de déterrer le corps. Elles passent ensuite du temps à l'entrée du cimetière à chanter le lignage du défunt.

Certaines grandes initiations, sont un cadre privilégié, à la fois pour dérouler le répertoire des chants ancestraux et pour l'enseigner à ceux qui ne le connaissent pas. C'est ce que j'ai pu observer en 2011, lors de l'initiation royale appelée $E w a \eta^{9}$ organisée par le roi d'Oussouye.

8 Exemple : «Kakit éyya » que l'on peut traduire par : commencer à tisser un panier.

9 Cette initiation qui a lieu à peu près tous les trente ans, concerne les lignages les plus proches de la royauté d'Oussouye. Un roi n'organise qu'une seule initiation de ce type durant son règne. Il y a eu plus de deux milles initiés en 2011, dont la grande majorité était de la diaspora. 
D'autres événements moins formels donnent lieu à une exécution parfois spontanée du répertoire. C'est le cas lorsqu'un individu fait un exploit lors d'une séance de lutte, d'une chasse collective, d'un match de football, etc.

Par ailleurs, certaines fêtes chrétiennes ou musulmanes (baptême, mariage, communion) sont devenues de grands moments de chants surtout dans les grandes villes où vit un nombre important de Jóola. Il n'est pas rare qu'après avoir entendu de la musique moderne une bonne partie de la journée, certains réclament pour la fin, le chant kuhuluy comme un hommage à l'organisateur de la fête.

\section{Kubuluy, un chant « tissé » à partir d'éléments choisis}

La composition des chants kuhuluy est un travail très méticuleux. En parlant de tissage, je pense au parallèle qui peut être fait avec le pagne mortuaire qui est un autre élément essentiel des funérailles jóola et des échanges entre familles (Diatta 1998). L'on ne peut envisager un enterrement sans ce pagne que se doivent d'apporter certaines personnes en fonction des liens de parenté, d'alliance, ou d'amitié. Ce pagne, appelé kahul était autrefois tissé par les femmes, notamment dans le royaume voisin de Seleeki, à l'Est d'Oussouye ${ }^{10}$.

Lors d'un décès dans un lignage, les familles alliées et les amis du défunt apportent chacun un pagne funéraire $(\mathrm{kahul})$ comme participation à l'ensevelissement du mort. Cette démarche est appelée en Jóola kahok c'est-à-dire « enterrer ». L'acte de donner un pagne et celui de mettre en terre sont considérés comme étant de même valeur. Souvent, le nombre très important de pagnes ne permet pas de tous les déposer dans le cercueil. Toutefois, certains pagnes doivent nécessairement et prioritairement être placés au plus près du corps. L'ordre suivi est généralement le suivant : d'abord le pagne d'un des époux pour ensevelir son conjoint, ensuite celui du patrilignage, du matrilignage, de l'ami intime et ainsi de suite. Une dizaine de pagnes voire plus peuvent servir à envelopper le corps. La valeur accordée par la communauté à un défunt est relative au nombre de pagnes réunis et au nombre de bovins immolés pour ses obsèques. Un proverbe dit à ce propos : «Atumeta, jilees dahoomei » : « la mauvaise langue, on l'enterre avec un petit pagne ».

Ce proverbe, commente Nazaire Diatta, fait allusion à l'importance attachée à de belles funérailles. Le sérieux avec lequel la danse est faite donne le cofficient d'estime de celui qui s'est éteint. Il en est de même du nombre de pagnes utilisés pour l'inhumation. (Diatta 1998 : 47)

Le reste des pagnes qui n'aura pas servi à ensevelir le corps est gardé dans un même vase en argile. Chaque lignage possède une réserve de ce genre et c'est dans ce vase, toujours bien conservé chez le plus ancien du patrilignage, que sont prélevés des pagnes lorsque la famille en question doit à son tour présenter des condoléances ailleurs.

Couvrir le corps d'un défunt du pagne funéraire (kahul) est appelé kahuluyen. Cette disposition est un droit pour toute personne. Ensevelir le corps avec un pagne est une obligation majeure. Lorsque par exemple une personne découvre une dépouille non identifiée, il revient à sa famille de tirer de ses réserves les pagnes nécessaires

10 Actuellement ces pagnes sont achetés dans le marché (industriel) ou auprès d'artisans tisserands, généralement des hommes de l'ethnie Manjak installés dans des villes comme Ziguinchor. 
pour enterrer dignement l'inconnu. Ne pas respecter cette disposition expose à des sanctions sévères de la part de l'autel royal Eluy qui garantit l'observance par tous des lois communautaires.

Exception est faite cependant pour certains types de personnes dont la société simplifie drastiquement les obsèques. C'est le cas généralement pour des personnes nées avec de lourds handicaps, les lépreux ou les enfants inscrits dans une série de décès successifs dans la même fratrie. Dans ce dernier cas par exemple, l'enfant qui décède est considéré comme étant le même qui revient constamment à la vie dans la même famille et qui ne cherche en réalité qu'à s'enrichir des dons notamment de pagnes qui lui sont faits à ses funérailles. Pour interrompre ses allers et venues, ses obsèques sont chahutées et son inhumation est faite sans pagne funéraire digne ${ }^{11}$. Ceux dont les obsèques sont ainsi «bâclées " sont enterrés dans un coin spécial du cimetière et ne bénéficient pas de chants ancestraux.

Je reviens au terme kahuluyen qui, en plus de signifier le fait de recouvrir le corps avec un pagne funéraire, traduit également l'interdit de prononcer le nom du défunt et son remplacement par le chant kuhuluy. Cette proximité terminologique permet de faire un lien entre le pagne funéraire et le chant. Les deux ont pour fonction de « couvrir le corps ». Le répertoire de chants funéraires serait, pour reprendre la belle expression de Jean-Pierre Warnier, une « enveloppe sonore » (Warnier $2009: 64$ ) qui permet de ne garder en souvenir que les belles actions du défunt.

Couvrir, recouvrir, envelopper, sont des gestes essentiels dans le processus jóola d'ancestralisation. Les développements de Didier Anzieu dans « Le moi peau » (Anzieu 1985) sur la fonction de la peau dans les rapports entre l'individu et son environnement social immédiat, me paraissent éclairants ici. Selon Anzieu la peau remplit à la fois trois fonctions : comme sac, comme surface entre le dedans et le dehors, et comme zone d'échange et de communication.

L'ensevelissement avec les pagnes funéraires tout comme les chants qui enveloppent la mémoire du défunt marquent radicalement son individualité jusque dans la tombe ${ }^{12}$. Il en est de même des surnoms utilisés pour composer les chants, qui sont les traces d'un parcours unique.

La couverture du défunt est aussi l'expression d'un lieu de contact entre le dedans et le dehors. La diversité de la provenance des pagnes (famille, alliés et amis) ou des voix qui composent les chants, montrent le lien entre le défunt et son environnement social. De ce point de vue, la « couverture » peut être considérée comme un renforcement de la protection de l'individu contre toute agression.

Physiquement, le corps mis en terre est bien protégé et n'entre pas en contact direct avec la terre grâce aux pagnes. Le réajustement de la tombe par les femmes, le lendemain de l'enterrement pourrait être compris dans cette même logique de bien recouvrir le corps. Socialement, l'interdit de prononcer le nom du défunt empêche toute prise sur lui. Ne restent que les chants louangeurs qui invitent à ne retenir que le positif

11 Les mères dont les enfants meurent de façon successive suivent un rite appelé Kañalen, voir Odile Journet-Diallo 1981, 1985, 2001, 2007.

12 Chez les Jóola, une tombe ne doit contenir qu'un seul corps. Ce principe explique que lorsqu'une femme meurt en état de grossesse il est procédé à une extraction du fœtus pour que les deux corps soient enterrés séparément. 
et à garder une certaine forme de communication avec le défunt. De cette protection sociale sont exclus ceux dont le retour n'est pas souhaité par la collectivité et qui sont exclus du processus d'ancestralisation. Leur mort physique est comme redoublée par une mort sociale, sans traces (Vidal $1986: 18$ ).

Le parallèle pagne/chant kuhuluy peut être poussé plus loin en les considérant comme deux réserves destinées aux deux parties du lignage : la partie trépassée et celle vivante. Les pagnes intègrent le défunt à la portion de terre du lignage au niveau du cimetière tandis que le chant kuhuluy l'associe au répertoire lignager, gardé et utilisé par les vivants dans les rapports sociaux.

\section{Le chant kubuluy comme moyen de légitimation}

L'usage du répertoire de chants ancestraux peut servir comme moyen d'action dans les rapports de pouvoir au sein d'une collectivité. J'ai parlé précédemment de la diversité des occasions qui président à la mobilisation de ce répertoire. Je reprends ici l'exemple d'un lutteur qui fait une performance en venant à bout d'un adversaire redoutable. Il est demandé dans pareille circonstance que soit entonné le répertoire auquel il est rattaché, à savoir celui de son patrilignage. L'expression pour le dire est : «jikitul ool », voulant dire littéralement « commencez pour lui ». L'enchaînement des chants partira de l'ascendance directe du concerné pour remonter vers l'ensemble du lignage et au besoin des lignages cousins et ceux du village.

Je retiens deux aspects qui me paraissent d'un grand intérêt pour le sujet : 1) la dimension musicale et 2) la dimension généalogique du répertoire.

Dans un article sur la musique et l'organisation sociale chez les Indiens Suyá du Mato Grosso (Brésil) Seeger Anthony remarque que :

la musique est plus qu'un simple domaine auquel un anthropologue pourrait librement choisir de s'intéresser ou non; il s'agit d'un domaine riche en enseignements, y compris dans la vision que les anthropologues ont des sociétés humaines. (Seeger $2004: 148$ )

Chanter est en effet une forme « d'habillage de la parole » (Brandly 2004 : 308) qui donne à la communication et aux interactions une dimension particulière. Exécuté en groupe, le chant en lui-même a une force de cohésion importante. Et chanter en groupe les mêmes chants peut être ressenti comme un acte d'union, une enveloppe sonore dans laquelle évoluent des sujets synchronisés sur le tempo et la pulsion du chant (Warnier 2009 : 64).

Par ailleurs, le village qui chante les chants ancestraux de son lutteur ingénieux, reconnaît de fait l'inscription de celui-ci dans l'ensemble villageois (qu'il honore par ses prouesses) et se trouve en quelque sorte tenue de reconnaître cette légitimité en chantant. Ainsi donc, par le chant, la communauté légitime l'ascendant et le descendant.

Le principe d'exécution du chant kuhuluy est, je le rappelle, de parcourir un répertoire impliquant une ascendance en passant d'un ancêtre à un autre. Il n'est jamais question de n'entonner que les chants d'un seul ancêtre. La manière dont sont déclamées les chansons est faite pour passer d'une personne à une autre. Chaque chant est en effet repris deux à trois fois entre deux chœurs qui se répondent alternativement. Celui qui est chargé d'introduire les chants remonte ainsi la généalogie. 
Il n'y a pas de griot chez les Jóola tel qu'on peut le voir dans d'autres sociétés voisines au Sénégal ou ailleurs. Mais en réalité, le rôle joué par le griot dans les sociétés qui en possèdent est ici accompli par le groupe lui-même, à savoir le village ou le lignage. Je conviens alors avec Mamoussé Diagne que :

le griot généalogiste, qui déclame la noble lignée de quelqu'un, entend dire à la société, dans le même mouvement, la reconnaissance d'un statut et le rappel du système classificatoire qui le légitime. Mais il s'adresse prioritairement à la personne même dont la place se trouve ainsi confirmée. À l'occasion de grandes épreuves, le simple rappel de la généalogie qui constitue le noyau de l'éloge trace la voie à suivre, en insufflant une énergie supérieure. (Diagne 2005 : 365)

Dans le chant kuhuluy la communauté qui chante peut s'adresser à plusieurs acteurs en même temps : l'individu concerné, la communauté elle-même et les villages voisins. À celui dont l'ascendance est chantée, il est rappelé ses origines et par conséquent sa place au sein de la collectivité. Le chant est sous cet angle un acte d'institution au sens où l'entend Bourdieu :

\begin{abstract}
L'acte d'institution est un acte de communication mais d'une espèce particulière : il signifie à quelqu'un son identité, mais au sens à la fois où il la lui exprime et la lui impose en l'exprimant à la face de tous (katègoresthai), c'est, à l'origine, accuser publiquement) et en lui notifiant ainsi avec autorité ce qu'il est et ce $q u$ 'il a à être [...] C'est par l'intermédiaire de l'effet d'assignation statutaire ( "noblesse oblige ») que le rituel d'institution produit ses effets les plus « réels»: celui qui est institué se sent sommé d'être conforme à sa définition, à la hauteur de sa fonction. L'héritier désigné - selon un critère plus ou moins arbitraire-est reconnu et traité comme tel par tout le groupe, et d'abord par sa famille, et ce traitement différent et distinctif ne peut que l'encourager à réaliser son essence, à vivre conformément à sa nature sociale. (Bourdieu $1982: 60$ )
\end{abstract}

Le « deviens ce que tu es », qui, comme le dit Bourdieu, est la formule qui soustend toute la magie performative des actes d'institution (1982:61) est particulièrement perceptible lors des danses funéraires. Généralement, lorsque l'on chante les ancêtres d'un lignage, quelques membres du lignage chanté se détachent de la danse pour exalter chacun ses qualités soit de bon lutteur, de bon cultivateur, d'intellectuel, etc. Les hommes sont fortement poussés à ces formes de glorification par leurs agnates qui les accompagnent durant ces mises en scène, comme pour confirmer leurs dires et signifier que les ancêtres n'ont pas à désespérer de leur descendance.

En chantant, la communauté s'adresse aussi à elle-même et reconnaît être constituée d'une diversité de qualités qui font son indépendance et la garantie de sa survie. Chanter c'est accepter le discours qui est tenu sur un individu, un lignage et un village.

Les chants héroïques dont regorge le répertoire des ancêtres sont aussi comme un clin d'œil au village voisin au dépend de qui les héros en question ont acquis leur gloire. Nazaire Diatta a bien raison de dire que dans beaucoup de cas, ces chants ne jouent pas en faveur de l'entente. Ils relatent une situation de conflit et par les armes et par le travail, la richesse. Dans la danse funéraire, par les chants, un village trouve plaisir à rouvrir les plaies, les conflits, dans une volonté d'affirmation, en opposition à l'autre (Diatta $1982: 50$ ). 
Par le chant généalogique, destin individuel et destin de groupe s'articulent pour délimiter un espace qui est celui de la normativité sociale et de la circulation symbolique. Prendre à témoin les morts et les vivants qui peuplent cet espace, c'est s'inscrire dans la quête de reconnaissance qui habite toute revendication de légitimité (Diagne 2005 : 364-365).

\section{Et comme l'écrit Diagne,}

Si être, c'est être reconnu dans un jeu complexe de positions et de statuts, on tient là la preuve indubitable que le recours à l'évocation généalogique ne se tourne vers le passé que pour établir une place dans le présent, et/ou une projection programmatique en direction du futur. Ce qu'elle met en premier plan, c'est toujours la question du pouvoir posée, non pas sous l'angle de la légalité (du pouvoir de fait), mais sous celle de la légitimité (de l'ayant droit au pouvoir) [...] On peut risquer l'hypothèse selon laquelle la récapitulation généalogique est une manière, pour une société orale, de procéder à une mise en scène de l'occupation de son territoire. Cadastre symbolique pour l'essentiel, où se donne à lire les droits sur certains lieux et certaines choses, que peuvent exhiber à tout moment les héritiers, à condition de se tenir prêts à en renouveler le bail. (Diagne $2005: 365-366$ )

La question de la légitimation par le chant chez les Jóola et l'occupation territoriale qu'elle sous entend peut se comprendre à la lumière des analyse de Michel Izard à propos de la construction d'un État dans le Yatenga. Michel Izard remarque que l'appareil d'État émerge d'une double procédure, l'une disjonctive et l'autre conjonctive.

L'État, né de la conquête, n'existe en tant que tel que pour autant que la conquête est niée, et le discours de l'histoire a dès lors pour fonction générale l'intériorisation idéologique de cette négation. (Izard 1992 : 53)

Cette double procédure de disjonction et de conjonction se retrouve dans la société jóola, d'abord au niveau lignager et ensuite au niveau du village. Un bref aperçu de la morphologie de ce qui est considéré comme lignage ou village en milieu jóola permet de mieux cerner la problématique. Les lignages, tels qu'ils se présentent aujourd'hui, sont de manière générale, le résultat d'une construction à partir d'éléments disparates. Plusieurs segments de lignages peuvent être associés autour d'un lignage souche (Journet-Diallo 2007) pour devenir un seul patrilignage ayant en commun divers autels dont celui des ancêtres et un répertoire commun de chants ancestraux. Diverses raisons justifient une telle configuration.

La guerre est une des raisons les plus couramment évoquée. En effet, les guerres inter-villageoises se soldaient souvent par la dispersion des vaincus et leur intégration au sein d'autres lignages dans les villages voisins ou même chez le vainqueur ${ }^{13}$. Dans d'autres cas, la raison est économique. L'insuffisance de terres cultivables oblige des individus à migrer vers d'autres localités où ils sont hébergés et intégrés. Ce peut être aussi des raisons rituelles qui amènent un lignage à s'agréger de nouveaux éléments

13 Les guerres jóola sont très codifiées. Les guerriers sont soumis à un code assez strict en termes de respect de la personne humaine, surtout de la femme, de l'enfant et du réfugié. Ce qui fait que les guerres faisaient en définitive plus de déplacés que de pertes en vie humaine (Journet-Diallo 2007). 
qui seront comme une réserve dans laquelle seront pris les desservants d'autels, la responsabilité rituelle étant généralement confiée aux nouveaux venus.

Quant au village jóola, il se construit en permanence à partir de ses unités qui s'emboîtent les unes dans les autres : le lignage dans le quartier et celui-ci dans le village. Cette organisation fait que le village est un ensemble avec des centres variables comme le remarque Odile Journet-Diallo :

Un village ne se forme pas à partir d'un centre mais uniquement à partir d'un emboîtement de relations espace central/périphérie, qui se démultiplient à chaque échelle considérée. (Journet-Diallo 2007 : 76)

Le travail d'intégration mobilise la communauté villageoise dans son ensemble et met en place des dispositions notamment symboliques pour l'encadrer au mieux. L'intégration des nouveaux venus est strictement régie par des interdits. Rappeler à quelqu'un son intégration récente ou encore son éventuelle situation servile le cas échéant, expose à des dédommagements auprès des autels ${ }^{14}$. Cependant, le rapport au sein du lignage entre souche ancienne (aînés) et souche récente (cadets) ne disparaît jamais définitivement. Il ressort notamment à l'occasion des initiations dans la disposition des novices et l'ordre d'entrée en forêt initiatique. La procédure disjonctive n'est alors jamais déclamée, mais plutôt mise en scène dans les rituels surtout dans l'ordre processionnaire ${ }^{15}$.

La procédure conjonctive est, quant à elle, proclamée à travers le chant, comme discours unifiant, qui fait plus qu'insérer un individu dans un lignage. Il n'est pas fait de différence entre aînés et cadets dans le déroulement du répertoire de chants. C'est plutôt l'événement qui dicte le point de départ. On commence toujours par les ancêtres de celui qui est à l'honneur.

Le rapport de forces entre aînés et cadets se situe dans cette tension entre le temps de la déclamation (qui peut être tout événement important) et le temps de la mise en scène des positions sociales (la place occupée dans les processions lors des grands rituels tels que les initiations). Une famille récemment installée et qui a donné des héros au village sera toujours chantée avec reconnaissance. Ce fait peut servir d'arguments à ses membres pour affirmer leur pleine appartenance à la collectivité. Mais il lui sera rappelé sa position de nouveau venu à l'occasion des rituels initiatiques où elle devra se positionner en fonction des familles plus anciennement installées. Le chant est un des lieux d'expression du village dans la diversité de ses composantes. Il est un cadre offert à chacun pour revendiquer une légitimité et reconnaître aussi la place qui est la sienne.

\section{Le chant kubuluy et le défi de la transmission}

Les funérailles sont le contexte " ordinaire » de transmission du répertoire des chants ancestraux. Le village est dans ce cas le cadre idéal, puisque les chants sont généralement appris à force de les entendre chanter et de s'y entraîner. Lorsque des jeunes présentent très tôt des qualités de chantre et de mémorisation, ils peuvent

14 Voir sur ce sujet le développement de Paul Diédhiou 2011 : 64-67.

15 Mettre en rang les novices est une des caractéristiques des grands rites initiatiques jóola. 
bénéficier d'un enseignement plus approfondi auprès d'un parent ou d'un membre de la communauté villageoise. Certaines grandes initiations telles que les circoncisions ou les initiations royales peuvent servir de cadre formel d'apprentissage. Mais l'écart très important entre les initiations (trente ans ou plus entre chacune) ne favorise pas une transmission continue.

Divers facteurs tels que les conversions aux religions universalistes, l'école, les migrations, etc. ont rendu la transmission de ces répertoires très difficile. La connaissance parfois parcellaire des chants par les jeunes générations compromet souvent l'animation de certaines cérémonies comme les veillées funèbres ou les danses funéraires.

Le facteur migratoire est sans doute celui qui complique davantage la transmission. La société jóola est en effet fortement marquée par la migration. La masse de la littérature scientifique sur ce sujet le montre bien (Mark 1977, 1985 ; Hamer 1981, 1983 ; Lambert 2002 ; Foucher 2002 ; Linares 2003). Comme le souligne Foucher, l'expérience migratoire a été tellement massive pour les Jóola, ses effets tellement profonds pour les migrants comme pour les « sédentaires », qu'il est raisonnable de penser que la constitution de l'identité " casamançaise/diola » doit beaucoup aux réflexions et aux luttes autour de cette expérience (Foucher 2005 : 437).

En Basse Casamance l'intensification des affrontements entre l'Armée sénégalaise et les indépendantistes du Mouvement des forces démocratiques de Casamance (MFDC) autour des années 1990 (Foucher 2002 ; Marut 2010) a fixé dans les villes sénégalaises, gambiennes et Bissau guinéennes, une bonne partie de ce qui n'était avant cela qu'une migration saisonnière. Actuellement dans les villages autour d'Oussouye par exemple, les communautés installées dans de grandes agglomérations comme Dakar sont plus importantes que celles qui sont au village. Ce que note Lambert (Lambert 2008) à propos d'un village jóola au nord de la Casamance est valable pour ceux dont il est question ici. Lambert remarque qu'à Mandégane, la migration est la norme, un fait de la vie compris comme tel et se passant d'explication. C'est plutôt la personne qui n'est pas ou n'a jamais été un migrant qui fait figure d'exception (Lambert 2008).

Ces fortes concentrations de ressortissants du même village, souvent regroupés au sein d'associations (Foucher 2005) sont de véritables extensions des villages d'origine des migrants. L'impact de la migration sur les sociétés jóola a permis à Lambert dans son ouvrage «Longing for Exile » (Lambert 2002) d'étudier la façon dont les migrants se réapproprient l'espace, non pas tant en termes d'adaptation à un nouveau lieu, mais plutôt comment la communauté elle-même s'élargissait pour englober de nouveaux lieux.

Cette perspective est intéressante parce qu'elle permet de voir comment finalement le village et ses problématiques sont démultipliés, chaque communauté essayant avec les moyens dont elle dispose de reproduire les rapports sociaux qu'imposent notamment les liens de parenté.

C'est dans ce contexte que les chants ancestraux sont redevenus, après un temps de relative négligence ${ }^{16}$, objet de beaucoup d'intérêt, particulièrement pour les jeunes

16 Les conversions au Christianisme ou à l'Islam expliquent en partie ce «creux » dans la production et la transmissions des chants ancestraux puisque toute cette phase de composition de chants n'a pas lieu lorsque c'est un chrétien ou un musulman qui meurt. 
générations. Ce regain d'intérêt pour les chants kuhuluy s'inscrit dans ce qui est appelé plus largement par les populations locales elles-mêmes « le retour aux sources ».

J'ai particulièrement remarqué cet intérêt en 2011 en observant entre février et octobre les différentes étapes de l'initiation royale Eway, dont la précédente édition s'était déroulée en 1962. Durant les deux mois qu'a duré la réclusion des novices dans la forêt royale à Oussouye, l'exécution du répertoire des chants ancestraux occupait une place importante. Deux ou trois fois par jour, on pouvait les entendre chantés, quelle que soit l'heure, les conditions climatiques, etc. À la fin de l'initiation, j'ai alors noté la grande fierté que beaucoup de jeunes retiraient du fait qu'ils connaissaient enfin assez bien les chants des rois et surtout les chants kuhuluy de leur famille et village. Un initié me disait alors : " tu verras maintenant que lorsqu'il y aura des funérailles à Oussouye, rien ne sera plus comme avant, nous connaissons bien les chants ».

Les nouveaux moyens de communication sont mis à contribution, principalement par ceux de la diaspora. Les grandes cérémonies sont enregistrées, filmées, gravées sur dvd et expédiées dans les villes sénégalaises, en Europe ou en Amérique. Ces moyens permettent aujourd'hui à la diaspora de garder active la mémoire des chants kuhuluy et de la mobiliser au besoin.

\section{Conclusion}

En partant de l'élaboration du chant des ancêtres à la diversité des circonstances de sa mobilisation, j'ai voulu d'abord montrer en quoi ce répertoire de chants est le fruit d'une action collective et ensuite comment dans les usages, il engage les différents acteurs, aussi bien ceux qui l'exécutent que ceux qui l'écoutent.

Il est manifeste que le chant des ancêtres est intimement lié au patrilignage et à la terre comme lieu de transit. C'est au cœur de ces liens de parenté, réels ou fictifs, que naît et s'élabore le chant, dans un « dialogue » entre agnats, avant qu'il ne soit appris à l'ensemble du village. L'intégration ou la reconnaissance de l'individu dans les liens familiaux, à la naissance (par la présentation à l'autel des ancêtres du nouveau-né) comme à la mort (avec la composition des chants), préside à son incorporation dans l'autre unité qu'est le village.

Le patrilignage et le village, en tant qu'unités rituelles et politiques, sont le cadre au sein duquel se joue l'évocation des ancêtres. Le patrilignage est toujours le point de départ de tout déroulement du répertoire et le village (l'ensemble des patrilignages) le point culminant. Chaque ancêtre devient ainsi une trace dans l'histoire du village, dont le rappel périodique mobilise les différents acteurs du jeu social.

Le répertoire de chants ancestraux est une ressource protéiforme qui participe des interactions des différents acteurs à divers niveaux (lignager, villageois, inter-villageois etc.). De ce point de vue, l'évocation des ancêtres ne peut être pensée de manière unilatérale comme la projection du pouvoir d'un groupe d'individus (les aînés par exemple) sur un autre (cadets) tel que le suggère Kopytoff (1971 : 137). Au contraire, elle donne à chacun les moyens de prendre part à une praxis sociale en constance mutation.

La conjonction dans la création, l'entretien et l'usage de ce répertoire de chants, du principe de descendance et du principe territorial (droit au sol, droit à une sépulture dans le « cercle » familial, droit à des chants), fait tenir ensemble le religieux et le politique 
qui ne peuvent ici être pensés séparément. Cela justifie à mon avis l'intérêt accru de ce répertoire chez les Jóola convertis aux autres religions et ceux de la diaspora. Le chant des ancêtres donne à chacun l'opportunité d'affirmer son enracinement sur la terre des ancêtres même lorsqu'il vit au loin ou qu'il se réclame d'une religion différente de celle de ses pères.

\section{Références citées}

AdLER, Alfred, 2000. Le pouvoir et l'interdit. Royauté et religion en Afrique noire. Paris : Albin Michel.

AnZIEU, Didier, 1985. Le moi peau. Paris : Dunod.

Balandier, Georges, 1969. Anthropologie politique. Paris : PUF.

Baum, Robert, M., 1999. Shrines of the Slave-Trade : Diola Religion and Society in Precolonial Senegambia. Oxford : Oxford University Press.

Bourdieu, Pierre, 1982. Les rites comme actes d'institution, Actes de la recherche en sciences sociales, 43, pp. 58-63.

Brain, James L., 1975. « Ancestors as Elders in Africa - Further Thoughts », Africa, 41 (2), pp. 122-133.

BrandiLy, Monique, 2004. « Dire ou chanter ? L'exemple du Tibesti (Tchad)», L'Homme, 171-172, pp. 303311.

Calhoun, C. J., 1980. « The Authority of Ancestors : A Sociological Reconsideration of Fortes's Tallensi in Response to Fortes's Critics », Man (n.s.), 16, pp. 304-319.

Cormier-Salem, M-C., J.-P. Chauveau et S. Bouju (éds), 1999. Rivières du Sud. Sociétés et mangroves ouestafricaines. Paris : Éditions de L'IRD.

Diagne Mamoussé, 2005. Critique de la raison orale. Les pratiques discursives en Afrique noire. Paris : Karthala.

DiatTa, Nazaire, 1982. «Anthropologie et herméneutique des rites jóola : funérailles, initiations ». Thèse de Doctorat, Paris : EHESS.

-, 1998. Proverbes jóola de Casamance. Paris : Karthala.

DiéDHIou, Paul, 2011. L'identité Jóola en question (Casamance). La bataille idéologique du MFDC pour l'indépendance, pp. 64-67. Paris : Karthala.

ForTes, Meyer, 1959. Oedipus and Job in West African Religion. Cambridge : Cambridge University Press.

FOUCHER, Vincent, 2002. «Les « évolués », la migration, l'école : pour une nouvelle interprétation de la naissance du nationalisme casamançais », in Momar C. Diop, (dir.), Le Sénégal contemporain. Paris : Karthala, pp. 375-424.

-, 2005. « Les relations hommes-femmes et la formation de l'identité casamançaise », Cahiers d'Études africaines 178, (45), pp. 431-455.

GaBle, Eric, 1996. « Women, Ancestors, and Alterity among the Manjaco of Guinea-Bissau », Journal of Religion in Africa, 26 (2), pp. 104-121.

GIRARD, Jean, 1969. Genèse du pouvoir charismatique en Basse Casamance. Dakar : IFAN.

Hamer, A., 1981. «Diola Women and Migration : A Case Study », in Lucie Colvin (éd.), The Uprooted of the Western Sahel, pp. 183-203. New York : Prager.

-, 1983. « Tradition and Change : A Social History of Diola Women (Southwest Senegal) in the Twentieth Century ». Ph.D. dissertation, University of Michigan, Ann Arbor.

IzARD, Michel, 1992. L'odyssée du pouvoir. Un royaume africain : État, société, destin individuel. Paris : Éditions de l'EHESS. 
JindRA, M. et J. NoREt (éds), 2011. Funerals in Africa. Explorations of a Social Phenomenon. New York et Oxford : Berghahn.

Journet-Diallo, Odile, 1981. «Quête de l'enfant. Représentation de la maternité et rituels de stérilité dans la société jóola de Basse Casamance », Journal de la Société des Africanistes, 5, pp. 97-115.

-, 1985. « Les Hyper-mères n'ont plus d'enfants. Maternité et ordre social chez les Jóola de Basse Casamance », in N.C. Matthieu, L'arraisonnement des femmes. Paris : Éditions de l'EHESS «Cahiers de l'Homme», 24, pp. 17-36.

-, 2001. « Noms d'ancêtres, noms d'amis, noms de dérision ». Exemples africains, Spirale, 2001/3, 19, pp. 51-60.

-, 2007. Les créances de la terre. Chroniques du pays Jamaat (Jóola de Guinée-Bissau). Brepols : EPHE.

Kopytoff, Igor, 1971. «Ancestors as Elders in Africa », Africa, 41 (2), pp. 129-142.

LAMBert, Michael C., 2002a. "La marginalisation économique des communautés jóola à la fin du $20^{\text {ème }}$ siècle », in Momar C. Diop (dir. ), Le Sénégal contemporain. Paris : Karthala.

-, 2002b. Longing for Exile. Migration and the Making of a Translocal Community in Senegal, West Africa. Portsmouth : Heinemann.

-, 2008. "Réflexions sur le multilocalisme et les migrations internationales au sud du Sénégal et ailleurs », REVUE Asylon(s), 3, mars 2008, Migrations et Sénégal, url de référence :

$<$ http://www.reseau-terra.eu/article711.html $>$

Linares, Olga F., 2003. « Going to the City ... and Coming Back ? Turnaround Migration among the Joola of Senegal », Africa, 73 (1), pp. 113-132.

MARK, Peter, 1985. A Cultural, Economic and Religious History of the Basse-Casamance. Stuttgart : Franz Steiner Verlag.

-, 1977. « The Rubber and Palm Produce Trades and the Islamization of the Diola of Boulouf (Casamance, 1890-1920 », Bulletin de l'IFAN, 39, Série B, nº 2, pp. 341-361.

Marut, Jean-Claude, 2010. Le conflit de Casamance. Ce que disent les armes. Paris : Karthala.

McCALL, John C., 1995. «Rethinking Ancestors in Africa », Africa, 65 (2), pp. 256-270.

MÉGuelle, Philippe, 2012, Chefferie coloniale et égalitarisme diola. Les difficultés de la politique indigène de la France en Basse-Casamance (Sénégal), 1828-1923. Paris : L'Harmattan.

Mendonsa, Eugene L. 1976. « Elders, Office-Holders and Ancestors among the Sisala of Northern Ghana », Africa, 46 (1), pp. 57-65.

PéLISSIER, Paul, 1966. Les paysans du Sénégal. Paris : Imprimerie Fabrègue.

SeEger, Anthony, 2004. « 'Chanter l'identité'. Musique et organisation sociale chez les Indiens Suyá du Mato Grosso (Brésil) », L’Homme, 171-172, pp. 135-150.

THомаs, Louis V., 1959. Les Diola. Essai d'analyse fonctionnelle sur une population de Basse Casamance. Dakar : IFAN.

Tomas, Jordi, 2005. « La Identitat Ėtnica entre els Joola d'Oussouye (Hùluf Bubajum Àai) ». Thèse de Doctorat, Universitat Autònoma de Barcelona.

Tonda, Joseph, 2000. « Enjeux du deuil et négociation des rapports sociaux de sexe au Congo », Cahiers d'Études africaines, 157, pp. 5-24.

VIDAL, Claudine, 1986. «Funérailles et conflit social en Côte d’Ivoire », Politique africaine, 24, pp. 9-19.

WARNIER, Jean-Pierre, 2009. Régner au Cameroun. Le Roi-Pot. Paris : Karthala. 